\title{
A Comparison of Pitfalls after ALPPS Stage 1 or Portal Vein Embolization in Small-for-Size Setting Hepatectomies
}

\author{
Andreas A. Schnitzbauer \\ Clinic for General and Visceral Surgery, University Hospital Frankfurt, Goethe-University Frankfurt/Main, Frankfurt/M., Germany
}

Keywords

ALPPS · Portal vein embolization, PVE · Liver resection . Small-for-size setting · Pre-completion pitfalls · Failure

\section{Summary}

Background: Portal vein embolization (PVE) followed by resection and associating liver partition and portal vein ligation for staged hepatectomy (ALPPS) are tools to enable liver resections in small-for-size settings. Methods: A systematic review of the literature and comparison of pitfalls between PVE and resection and after ALPPS stage 1 were performed. Results: Evidence levels were as low as 4 for both procedures. 20 publications were identified with reports on post-PVE or post-ALPPS stage 1 pitfalls. A total of 2,758 patients treated with PVE followed by resection and 698 patients undergoing ALPPS were analyzed. Pitfalls identified were failure to advance to resection (PVE: high (20\%)/ALPPS: low (1\%); p = 0.0001), tumor progression (PVE: high/ALPPS: low); insufficient hypertrophy (PVE: frequent/ALPPS: rare), and inter-stage liver failure (PVE: rare/ALPPS: frequent). However, in-house mortality was still very high after ALPPS ( 7 vs. $3 \%, p=0.0001$ ) in a pooled analysis. Conclusion: PVE is a well-established technique to induce hypertrophy in small-for-size settings. The weakness of PVE is that it may fail to advance to resection. Inter-stage liver failure in ALPPS triggers post-stage 2 mortality. Prolongation of the inter-stage interval to overcome liver failure or cancellation of the resection stage combined with adherence to defined indications has the potential to make ALPPS much safer and decrease mortality rates. Level of evidence is low for both techniques.

(c) 2017 S. Karger GmbH, Freiburg

\section{Introduction}

Portal vein embolization (PVE) was first described by Kinoshita et al. [1] and Makuuchi et al. [2] more than 30 years ago. Its establishment in modern liver surgery allowed surgeons to perform safe resections in small-for-size settings (surgically possible but functionally not) where the future liver remnant (FLR) increases in size in response to PVE and the patient does not show signs of disease progression. With the interventional embolization or surgical ligation of the portal vein, the FLR is stimulated, obtaining growth rates of $25-50 \%$ within $4-6$ weeks [3]. In a second step, the tumor may be resected safely without driving patients into fatal post-hepatectomy liver failure, especially after extensive pre-resection chemotherapy [4, 5]. Until recently, PVE was an absolute sovereign and a monopolist with regard to enlarging the FLR. However, only 5 years ago, the group of Hans Schlitt established a new technique of 2-stage hepatectomy to induce FLR growth. The new technique combined portal vein ligation and parenchymal transection during the first stage of surgery, a short interval for FLR growth and regeneration (10 days), and the removal of tumor-bearing liver in the second step of surgery. The kinetic growth rates of the FLR were around $75 \%$ within $8-12$ days after stage 1 surgery and were larger than any other report so far $[6,7]$. Clavien and de Santibañes [8] established a now commonly used descriptive name for this new procedure: associating liver partition and portal vein ligation for staged hepatectomy, the so-called ALPPS procedure. However, just as most innovations suffer from initial throwbacks, the exploration phase of ALPPS also unveiled relevant morbidity in patients as well as unacceptable high mortality rates, especially in unselected patient cohorts and in certain indications such as perihilar cholangiocarcinoma [9-11]. Driven by the ALPPS registry, certain recommendations have been made concerning indications, surgical-technical modifications, and patient management, discussed and worded during the 1st international ALPPS consensus meeting in

\section{KARGER}

() 2017 S. Karger GmbH, Freiburg

Fax +497614520714
Prof. Andreas A. Schnitzbauer, MD, FACS, FEBS 

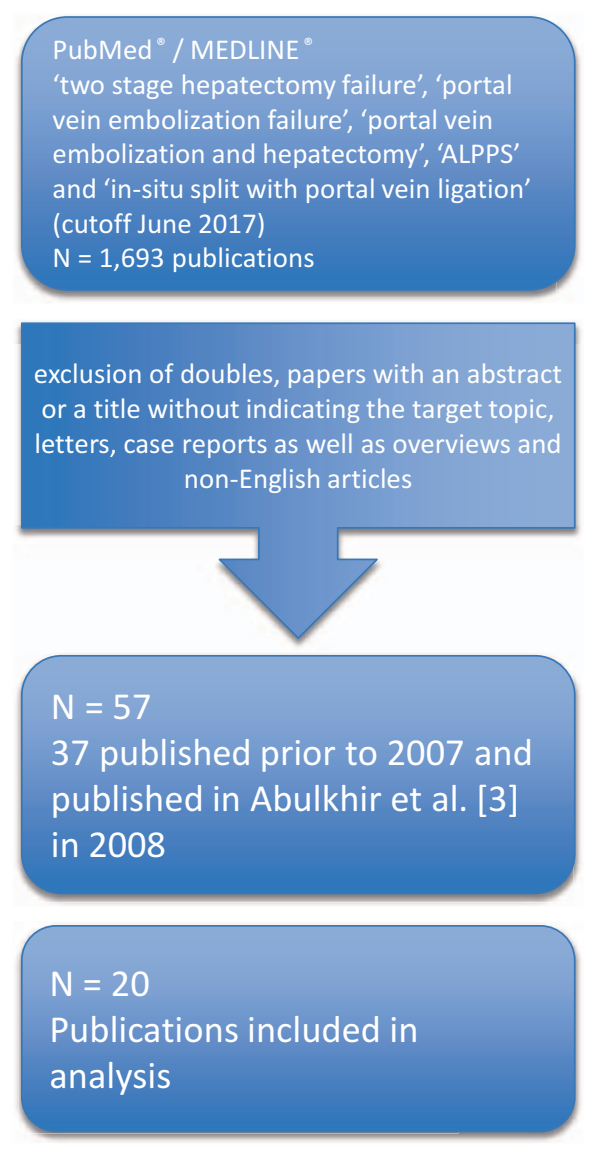

Fig. 1. Flow chart of paper selection.

Hamburg in 2015 [12]. Nonetheless, the evidence level for ALPPS benefit is still low, with no prospective randomized controlled trials comparing PVE and ALPPS so far. Besides, blinded by the $100 \%$ feasibility rate and the tremendous rates of hypertrophy, almost nobody focused on what happened between stages 1 and 2 of ALPPS. This time period, in which the critical pathophysiological damage occurs, is very likely to determine the further course of patients after ALPPS stage 2. Therefore, a systematic review was performed to detect and compare the pitfalls occurring in the interval between PVE and subsequent resection and between ALPPS stage 1 and 2 .

\section{Methods}

A PubMed/MEDLINE search was performed for the search terms '2-stage hepatectomy failure', 'portal vein embolization failure', 'portal vein embolization and hepatectomy', 'ALPPS', and 'insitu split with portal vein ligation'. Doubles and non-suitable articles were excluded. The search was performed 2 times to minimize the risk of selection bias. Articles were included in the analysis when the abstract reported failure rates, complications, or pitfalls after PVE or ALPPS stage 1. In more detail, target information was extracted focusing on inter-stage pitfalls that predicted liver failure after stage 2 in ALPPS, and post-PVE factors hindering progress to subsequent resection. Failure in PVE was defined as an event that did not allow advancement to resection, and in the ALPPS procedure, as critical factors that occurred between stages 1 and 2, discouraging advancement to stage 2 . Data were presented in a descriptive manner using total numbers, proportions, and risk factors where available. A cumulative pooled outcome analysis of patients was performed to analyze failure rate and in-house mortality. Evidence levels for benefit in both PVE and ALPPS were determined using the latest Oxford Centre of Evidence-Based Medicine recommendations [13]. Statistical analyses were performed with Review Manager (RevMan) Version 5.2. (The Nordic Cochrane Centre, The Cochrane Collaboration, Copenhagen, 2012).

\section{Results}

\section{Number of Publications and Selection}

A total of 1,693 publications were detected with the search terms described above until the cut-off date of June 2017. After exclusion of doubles, papers with an abstract or title not indicating the target topic, letters, case reports, as well as overviews and nonEnglish articles, 57 papers remained. Of those, 37 were published before the year 2007 and were in large part analyzed in the metaanalysis by Abulkhir et al. in 2008 [3]. A total of 20 papers remained for analysis to compare ALPPS and PVE, focusing on pitfalls leading to failure of the intended treatment completion. Figure 1 displays a flowchart of the paper selection.

\section{Evidence Levels for the Publications Analyzed}

Evidence levels were generally low for the interventions dealt with in this review (PVE followed by resection or ALPPS). Of the 20 publications analyzed, 2 were systematic reviews of case series and case-control studies comparing the 2 techniques, 13 were case series, and 5 were case-control studies. There were no cohort studies. This accounted for an overall evidence level of 4 for both PVE and ALPPS, as the review by Abulkhir et al. [3] was not a metaanalysis of randomized controlled trials.

\section{General Observations}

Resection strategies using PVE or ALPPS are infrequently employed at a rate of approximately 8 times per year (range 3.2-15.6) in very selected high-volume hepatobiliary centers. Unfortunately, most centers do not provide the whole volume of their liver program. Therefore, the proportion of liver resections in small-for-size settings using ALPPS or PVE remains an educated guess. Nonetheless, the total numbers given above are likely to be representative.

\section{Pooled Outcome Analysis of Failure and In-House Mortality \\ Comparing ALPPS versus PVE Followed by Resection}

Strikingly, the failure rate of PVE was considerably higher than that of ALPPS (fig. 2). A total number of 555 patients could not be resected after PVE whereas 1,956 were resected. In comparison, only 8 patients did not proceed to ALPPS stage 2, while 726 patients completed the treatment successfully. The difference was highly significant $(\mathrm{p}=0.0001)$. However, the in-house mortality 


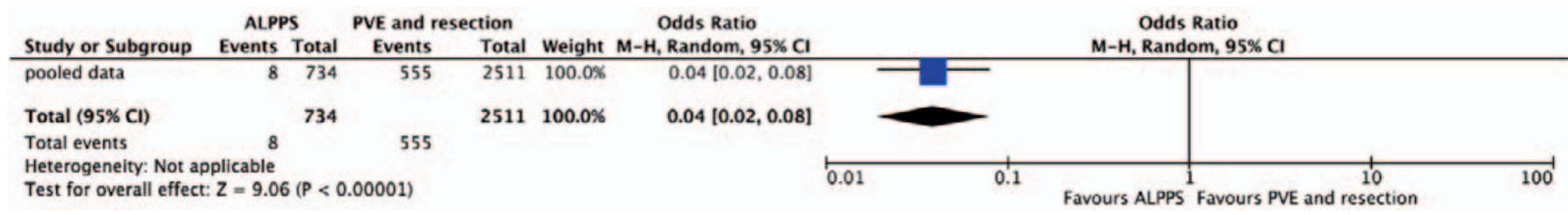

Fig. 2. Forrest plot of failure to progress to resection - ALPPS procedure compared to PVE followed by resection.

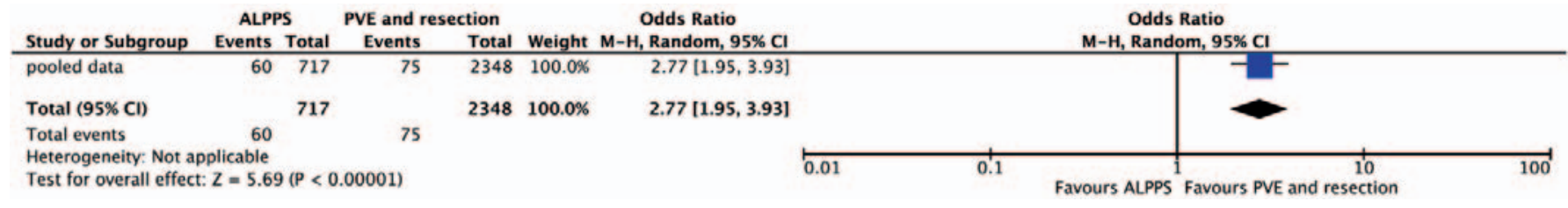

Fig. 3. Forrest plot of in-house mortality - ALPPS procedure compared to PVE followed by resection.

after PVE was significantly lower than that after ALPPS (fig. 3). Here, we see a completely different picture. After PVE and resection, only 75 patients died and 2,273 survived. In contrast, after the ALPPS procedure, 60 patients died compared to 657 survivors. This difference was again highly significant $(\mathrm{p}=0.0001)$.

\section{Pitfalls Detected after PVE Hindering the Advancement \\ to Resection}

In the systematic review by Abulkhir et al. [3], the overall morbidity after PVE (including post-resection complications) was very low with $2.2 \%$ of all cases. Insufficient hypertrophy after PVE and disease progression were the most frequent reasons why resection was not performed. Besides, other authors described a worsening of comorbidities during the waiting time after PVE and a misinterpretation of resectability as additional factors. However, there were also factors directly related to PVE; nausea/pain, thrombosis in the mesenterico-portal veins, esophageal varicose bleeding, liver hematoma, and incomplete or wrong embolization were serious albeit rare PVE complications that could be hazardous for the patients.

\section{Pitfalls Detected after ALPPS Stage 1 That Led to Adverse \\ Outcome after Stage 2}

Descriptions of inter-stage pitfalls after ALPPS remain rare. Truant et al. [14] performed the first systematic analysis. The authors described that the failure rate after ALPPS was 5\% due to insufficient growth, portal vein thrombosis, and inter-stage death, and that a delay in the planned procedure beyond day 9 was seen in more than $50 \%$ of patients. Schadde et al. [15] described that interstage liver failure and an inter-stage MELD score $>10$ were highly predictive for mortality after stage 2 . In detail, 21 of 28 patients with liver failure after stage 1 died within 90 days after stage 2, especially in the case of a MELD score $>10$ (odds ratio 3.9-4.9). Surgery-related fatal liver failure was finally confirmed in the publication by Linecker et al. [16]. Extracted data for all analyzed publications are displayed in table 1 . Table 2 summarizes the findings described above.

\section{Discussion}

The technique of PVE followed by resection was developed over several decades and is a well-established tool in small-for-size settings in liver surgery [1]. ALPPS, in contrast, is the new kid on the block. It was published only 5 years ago, and the first procedure was performed 10 years ago [6]. PVE followed by resection was developed within a considerable timeframe without competition from any other procedure, and was properly assessed. That way, data from long-term studies were obtained. ALPPS revealed certain flaws in its beginning but was developed properly by the introduction of a registry which made the procedure safer. Nonetheless, this review showed that pre-resection pitfalls remain poorly reported in both PVE and ALPPS, and that the evidence level is low with publications of predominantly level 4 [13]. Strikingly, the failure to advance to liver resection was rather high in patients with PVE. The reasons for PVE failure are well analyzed and consistently described throughout the literature; however, they still contribute to a failure rate of approximately $20 \%$ of all procedures. Reports on ALPPS failure after the stage 1 operation are still very rare. Manuscripts focusing on reasons for failure after ALPPS have so far only been published by Truant et al. [14], Schadde et al. [15], and Linecker et al. [16]. ALPPS still results in a very high in-house mortality and thus also impairs the oncologic outcome of the patients; however, mortality has somewhat improved with better patient selection along with the adherence to the recommended avoidance of certain indications such as perihilar cholangiocarcinoma $[11,17]$.

In this review, the overall mortality for the ALPPS procedure was $7 \%$ compared to $3 \%$ after PVE followed by liver resection. This demonstrates that the implementation of correct indications for ALPPS is pivotal. Additionally, we learn that inter-stage complications must lead to a reconsideration of whether to delay or even stop the progression to stage 2 . Since 21 of 28 patients with interstage liver failure died after completion of stage 2 , manifestation of liver failure (International Study Group of Liver Surgery [4]) after stage 1 should be regarded as a contraindication for completing 


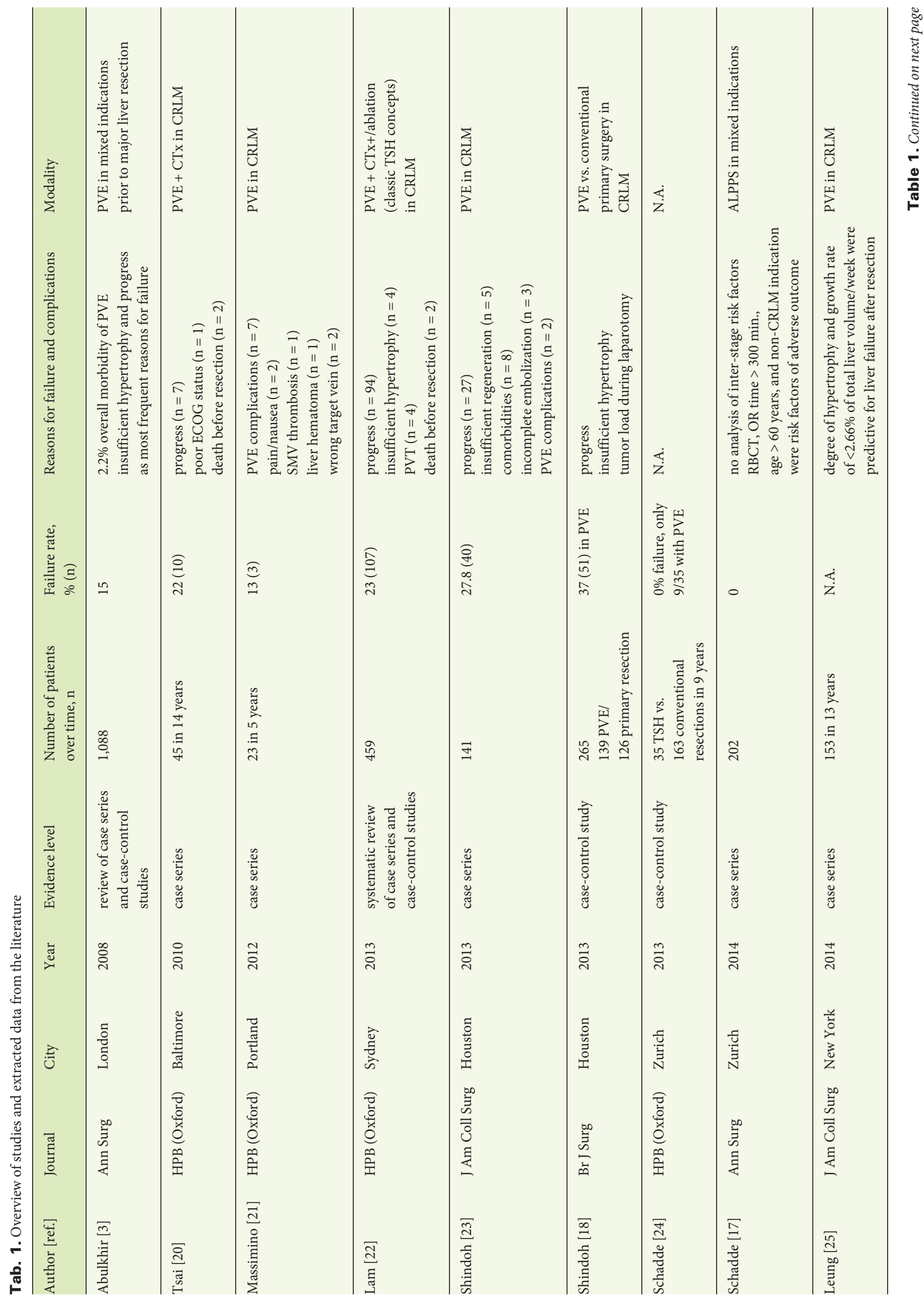




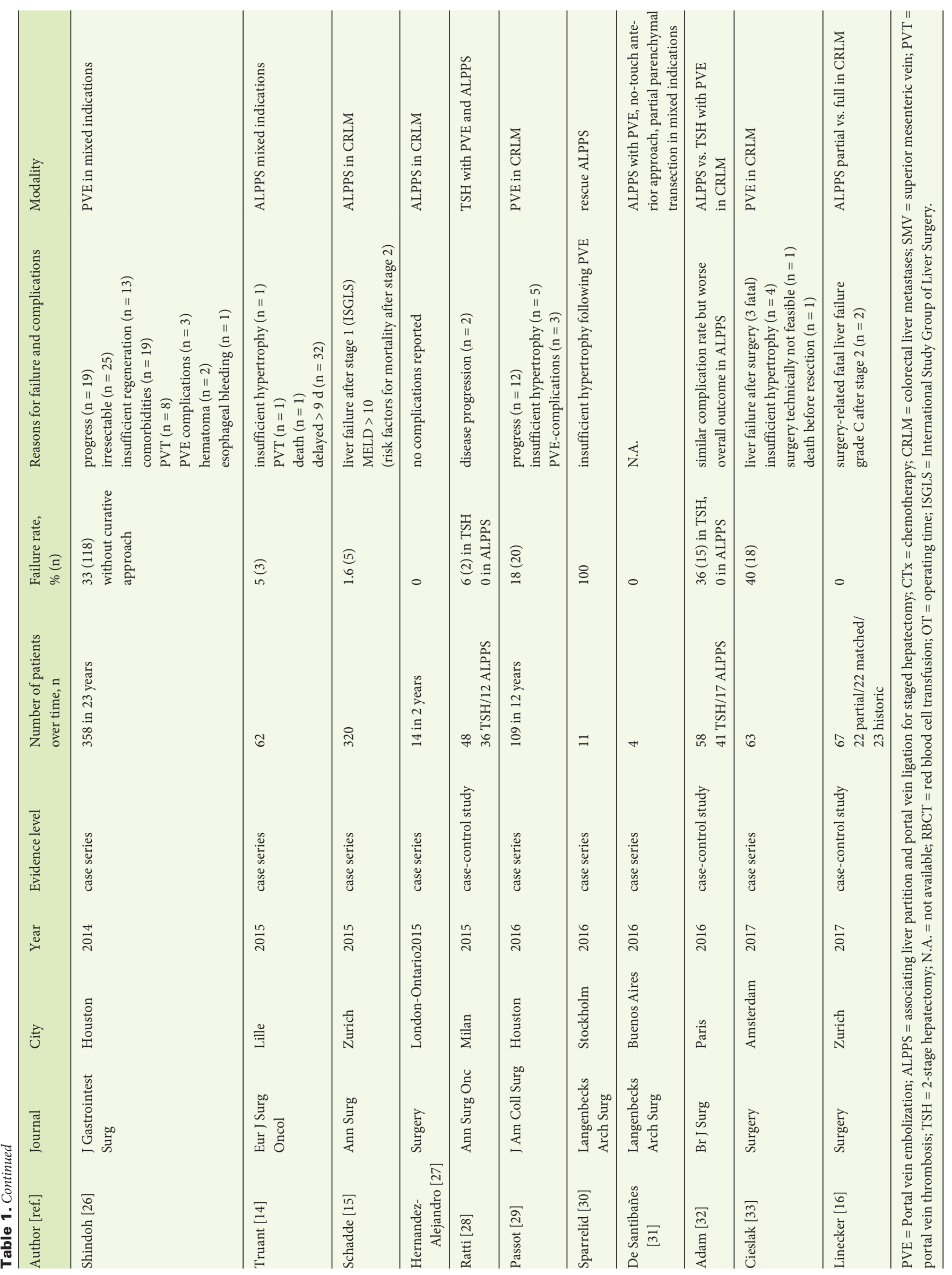


Tab. 2. Summary of key findings

\begin{tabular}{|c|c|c|c|}
\hline & $\begin{array}{l}\text { PVE and } \\
\text { resection }\end{array}$ & ALPPS & Conclusion/recommendations \\
\hline $\begin{array}{l}\text { Technique used, n } \\
\text { (over years) }\end{array}$ & $>30$ & $>10$ & $\begin{array}{l}\text { ALPPS data still in phase of exploration } \\
\text { (2b); however, recommendations indicate } \\
\text { entry into assessment phase (3) of surgical } \\
\text { innovations [34] }\end{array}$ \\
\hline Evidence level & level 4 (low) & level 4 (low) & $\begin{array}{l}\text { no randomized controlled trials, no direct } \\
\text { comparison of the } 2 \text { techniques available }\end{array}$ \\
\hline In-house mortality & acceptable & still too high & favoring PVE \\
\hline $\begin{array}{l}\text { Time interval } \\
\text { before final } \\
\text { resection }\end{array}$ & $2-4$ weeks & $7-15$ days & $\begin{array}{l}\text { favoring ALPPS; however, time interval can } \\
\text { be valuable for identifying patients that do } \\
\text { not profit from progressing to final proce- } \\
\text { dure for tumor biological reasons patients } \\
\text { with ALPPS inter-stage complications may } \\
\text { profit from a longer interval between stages }\end{array}$ \\
\hline $\begin{array}{l}\text { Failure rate } \\
\qquad \text { (no progression } \\
\text { to final resection) }\end{array}$ & very high & very low & favoring ALPPS \\
\hline Progress & frequent & rare & $\begin{array}{l}\text { may be a selection criterion and thus pre- } \\
\text { vent postsurgical in-house mortality; OS of } \\
\text { intention-to-treat analysis for PVE unclear }\end{array}$ \\
\hline PVE complications & rare & N.A. & $\mathrm{PVE}=$ established procedure \\
\hline $\begin{array}{l}\text { Insufficient } \\
\text { hypertrophy }\end{array}$ & frequent & rare & $\begin{array}{l}\text { favoring ALPPS, especially as rescue ALPPS } \\
\text { after failed PVE }\end{array}$ \\
\hline $\begin{array}{l}\text { Inter-stage } \\
\quad \text { liver failure }\end{array}$ & rare & frequent & $\begin{array}{l}\text { favoring PVE; may lead to delay of ALPPS } \\
\text { stage } 2 \text { making it a safer procedure }\end{array}$ \\
\hline Inter-stage death & rare & rare & not a common problem \\
\hline
\end{tabular}

PVE $=$ Portal vein embolization; ALPPS = associating liver partition and portal vein ligation for staged hepatectomy; OS = overall survival; N.A. = not available.

ALPPS as outlined by Schadde et al. [15]. The stage 2 operation can either be cancelled or be performed only in the case of recovery from liver failure. This has 2 advantages: First, the rate of liver failure-associated mortality would be reduced, and second, although potentially conflicting with the rapid hypertrophy principle of ALPPS, a longer waiting time could improve biologic selection. If the failure to advance to stage 2 increased from the currently reported rate of $1 \%$ to 5 or even $10 \%$, and if mortality rates were at the same time reduced to $3 \%$, ALPPS would have the potential be a more successful procedure than PVE. The overall number of patients with ALPPS failure would be much lower than that of those with PVE failure, resulting in better overall survival in an intention-to-treat setting due to the lower proportion of palliative treatment compared to patients with failure after PVE [18, 19].

The limitations of this analysis lie in the low level of evidence for both procedures. Double-checking all the included publications minimized the selection bias. However, the fact that only retrospective case series exist and the lack of head-to-head randomized controlled trials allowed only a descriptive comparison.
In conclusion, PVE is still the best option enabling safe liver resection in small-for-size settings. The pre-resection pitfalls after PVE are well described; however, failure to advance to liver resection after PVE leads $20 \%$ of patients to palliative treatment, resulting in a dramatic decrease in long-term oncologic survival. In patients with liver resection after PVE, however, the prognosis is still outstanding in this medically complex patient group. The outline of indications for ALPPS has much improved, but inter-stage problems are still poorly described. When inter-stage liver failure is avoided, the long-term prognosis after the ALPPS procedure is good. Either prolonging the inter-stage phase or cancelling the stage 2 procedure may allow the prevention of perioperative deaths in ALPPS, including an oncologically sound biologic selection.

\section{Disclosure Statement}

There are no conflicts of interest.

\section{References}

1 Kinoshita H, Sakai K, Hirohashi K, et al: Preoperative portal vein embolization for hepatocellular carcinoma. World J Surg 1986;10:803-808
2 Makuuchi M, Thai BL, Takayasu K, et al: Preoperative portal embolization to increase safety of major hepatectomy for hilar bile duct carcinoma: a preliminary report. Surgery 1990;107:521-527.
3 Abulkhir A, Limongelli P, Healey AJ, et al: Preoperative portal vein embolization for major liver resection: a meta-analysis. Ann Surg 2008;247:49-57. 
4 Rahbari NN, Garden OJ, Padbury R, et al: Posthepatectomy liver failure: a definition and grading by the International Study Group of Liver Surgery (ISGLS). Surgery 2011;149:713-724.

5 Vauthey J-N, Pawlik TM, Ribero D, et al: Chemotherapy regimen predicts steatohepatitis and an increase in 90-day mortality after surgery for hepatic colorectal metastases. J Clin Oncol 2006;24:2065-2072.

6 Schnitzbauer AA, Lang SA, Goessmann H, et al: Right portal vein ligation combined with in situ splitting induces rapid left lateral liver lobe hypertrophy enabling 2 -staged extended right hepatic resection in small-forsize settings. Ann Surg 2012;255:405-414.

7 Schadde E, Schnitzbauer AA, Tschuor C, et al: Systematic review and meta-analysis of feasibility, safety, and efficacy of a novel procedure: associating liver partition and portal vein ligation for staged hepatectomy. Ann Surg Oncol 2015;22:3109-3120.

8 De Santibañes E, Clavien P-A: Playing Play-Doh to prevent postoperative liver failure: the 'ALPPS' approach. Ann Surg 2012;255:415-417.

9 Oldhafer KJ, Donati M, Jenner RM, et al: ALPPS for patients with colorectal liver metastases: effective liver hypertrophy, but early tumor recurrence. World J Surg 2014;38:1504-1509.

10 Nadalin S, Capobianco I, Li J, et al: Indications and limits for associating liver partition and portal vein ligation for staged hepatectomy (ALPPS). Lessons learned from 15 cases at a single centre. Z Gastroenterol 2014;52:35-42.

11 Olthof PB, Coelen RJS, Wiggers JK, et al: High mortality after ALPPS for perihilar cholangiocarcinoma: case-control analysis including the first series from the international ALPPS registry. HPB (Oxford) 2017;19:381-387.

12 Oldhafer KJ, Stavrou GA, van Gulik TM, et al: ALPPS - where do we stand, where do we go?: eight recommendations from the first international expert meeting. Ann Surg 2016;263:839-841.

13 Howick J, Chalmers I, Glasziou P, et al: The $2011 \mathrm{Ox}-$ ford CEBM Levels of Evidence: Introductory Document. Oxford Centre for Evidence-Based Medicine, www.cebm.net/index.asp $x ? 0=5653$.
14 Truant S, Scatton O, Dokmak S, et al: Associating liver partition and portal vein ligation for staged hepatectomy (ALPPS): impact of the inter-stages course on morbi-mortality and implications for management. Eur J Surg Oncol 2015;41:674-682.

15 Schadde E, Raptis DA, Schnitzbauer AA, et al: Prediction of mortality after ALPPS stage-1: an analysis of 320 patients from the International ALPPS Registry. Ann Surg 2015;262:780-786.

16 Linecker M, Kambakamba P, Reiner CS, et al: How much liver needs to be transected in ALPPS? A translational study investigating the concept of less invasiveness. Surgery 2017;161:453-464.

17 Schadde E, Ardiles V, Robles-Campos R, et al: Early survival and safety of ALPPS: first report of the International ALPPS Registry. Ann Surg 2014;260:829-836, discussion 836-838.

18 Shindoh J, Tzeng C-WD, Aloia TA, et al: Portal vein embolization improves rate of resection of extensive colorectal liver metastases without worsening survival. Br J Surg 2013;100:1777-1783.

19 Folprecht G, Gruenberger T, Bechstein WO, et al: Tumour response and secondary resectability of colorectal liver metastases following neoadjuvant chemotherapy with cetuximab: the CELIM randomised phase 2 trial. Lancet Oncol 2010;11:38-47.

20 Tsai S, Marques HP, de Jong MC, et al: Two-stage strategy for patients with extensive bilateral colorectal liver metastases. HPB (Oxford) 2010;12:262-269.

21 Massimino KP, Kolbeck KJ, Enestvedt CK, et al: Safety and efficacy of preoperative right portal vein embolization in patients at risk for postoperative liver failure following major right hepatectomy. HPB (Oxford) 2012;14:14-19.

22 Lam VWT, Laurence JM, Johnston E, et al: A systematic review of two-stage hepatectomy in patients with initially unresectable colorectal liver metastases. HPB (Oxford) 2013;15:483-491.

23 Shindoh J, Vauthey J-N, Zimmitti G, et al: Analysis of the efficacy of portal vein embolization for patients with extensive liver malignancy and very low future liver remnant volume, including a comparison with the associating liver partition with portal vein ligation for staged hepatectomy approach. J Am Coll Surg 2013;217:126-133, discussion 133-134.
24 Schadde E, Slankamenac K, Breitenstein S, et al: Are two-stage hepatectomies associated with more complications than one-stage procedures? HPB (Oxford) 2013;15:411-417.

25 Leung U, Simpson AL, Araujo RLC, et al: Remnant growth rate after portal vein embolization is a good early predictor of post-hepatectomy liver failure. J Am Coll Surg 2014;219:620-630.

26 Shindoh J, Tzeng C-WD, Aloia TA, et al: Safety and efficacy of portal vein embolization before planned major or extended hepatectomy: an institutional experience of 358 patients. J Gastrointest Surg 2014;18:45-51.

27 Hernandez-Alejandro R, Bertens KA, Pineda-Solis K, et al: Can we improve the morbidity and mortality associated with the associating liver partition with portal vein ligation for staged hepatectomy (ALPPS) procedure in the management of colorectal liver metastases? Surgery 2015;157:194-201.

28 Ratti F, Schadde E, Masetti M, et al: Strategies to increase the resectability of patients with colorectal liver metastases: a multi-center case-match analysis of ALPPS and conventional two-stage hepatectomy. Ann Surg Oncol 2015;22:1933-1942.

29 Passot G, Chun YS, Kopetz SE, et al: Predictors of safety and efficacy of 2-stage hepatectomy for bilateral colorectal liver metastases. J Am Coll Surg 2016;223:99-108.

30 Sparrelid E, Gilg S, Brismar TB, et al: Rescue ALPPS is efficient and safe after failed portal vein occlusion in patients with colorectal liver metastases. Langenbecks Arch Surg 2017;402:69-75.

31 De Santibañes E, Alvarez FA, Ardiles V, et al: Inverting the ALPPS paradigm by minimizing first stage impact: the Mini-ALPPS technique. Langenbecks Arch Surg 2016;401:557-563.

32 Adam R, Imai K, Castro Benitez C, et al: Outcome after associating liver partition and portal vein ligation for staged hepatectomy and conventional two-stage hepatectomy for colorectal liver metastases. Br J Surg 2016; 103:1521-1529.

33 Cieslak KP, Huisman F, Bais T, et al: Future remnant liver function as predictive factor for the hypertrophy response after portal vein embolization. Surgery 2017; 162:37-47.

34 McCulloch P, Altman DG, Campbell WB, et al: No surgical innovation without evaluation: the IDEAL recommendations. Lancet 2009;374:1105-1112. 